\title{
Development of 18 DOF Salamander Robot Using CPG Based Locomotion for Straight Forward Walk
}

\author{
M. Munadi, Mochammad Ariyanto, Kharisma A. Pambudi, Joga D. Setiawan
}

\begin{abstract}
The utilization of mechatronics, robotics, and control systems has been widely spread in many areas over the last few decades. Robotics research is widely used for industrial purposes such as wheeled robots and manipulators, but nowadays many researchers have incorporated biomimetic science, often called biorobotics. This paper presents a preliminary research in the field of biorobotics with a robot salamander model that can walk in straight walking using a central pattern generator (CPG). This robot model uses a legged locomotion system that has 18 degrees of freedom (DOF). The CPG based locomotion model is developed for controlling the gait cycle when the robot walks. The motions of joint angle inputs resulted from CPG model is simulated using SimMechanics 3D Animation and implemented on the proposed salamander robot for straight walking. Based on the result in both virtual reality simulation and experimental work using CPG locomotion approach, the predefined joint angle inputs in salamander robot can be used to drive the robot. The proposed CPG locomotion can mimic the walking of a real salamander naturally in straight walk. Copyright (C) 2019 Praise Worthy Prize S.r.l. - All rights reserved.
\end{abstract}

Keywords: Robot Salamander, 18 DOF, Central Pattern Generator (CPG), 3D Animation

\section{Introduction}

When disasters such as flood, earthquake, tsunami, or landslide happen, people can be often found to be buried or trapped in an avalanche or ruin. In such a case, it is not possible to send people to enter the gaps or holes formed.

Therefore, it is necessary to develop a system that can solve this problem. One alternative is a robot that can enter the hole/slit and move on land or in water bringing the camera to see the dangerous conditions. Based on this condition, salamander robot is chosen because the salamander is one of the amphibian reptiles that can move both on land and on water. In this study, the proposed salamander like-robot model adapts the same robot that has been researched in previous studies [1]-[4].

It [1], [2], has 27 DOF: 11 DOF on the spine and four DOF on each leg [2]. It uses a servo motor as its actuator.

The legged locomotion of the salamander like-robot is developed by using central pattern generator (CPG) method. The salamander robot [1]-[4] has been developed using two types of locomotion: terrestrial locomotion to walk on the land and aquatic locomotion to swim on the water. In this research, the proposed salamander robot only uses terrestrial locomotion because the research study focuses on the motion of straight walk on a floor or land. The swimming mode of the salamander like-robot will be developed in the future study. CPG Based locomotion has inspired scientists and engineers to build robots by mimicking the spinal cord of the animals. CPG is the key mechanism of generating adaptive and versatile locomotion in animals [5].
CPG generates motion system instantly on a legged robot like spine based control system [6]. CPG based locomotion has been successfully implemented in legged robot research such as snake robot locomotion [6]-[9], hexapod robot [10]-[11], [19], robotic fish [5], [12]-[14], salamander robot [1]-[4], and quadruped robot [15], [20]. In this paper, the proposed of salamander like-robot uses a smaller number of DOF i.e. eight on the spine, three on each front leg and two on each rear leg. The simplification of the number of DOF is conducted to reduce the number of actuators so that the design becomes simpler and the salamander robot has a smaller dimension than the previously one as in [2]. This is conducted to meet the initial goal to create a salamander like-robot that is applicable for Search and Rescue (SAR) purpose. In this study, the governing equation of motion using a central pattern generator (CPG) has been developed for each robot on a straight walk. This paper proposes the CPG model of 18 DOF Salamanders likerobots by dividing the salamander robot body into smaller segments that have been performed by [1]. In straight walk locomotion, the CPG model can be driven by using a sinusoidal wave. The proposed CPG based locomotion of the spinal cord of the robot is represented by eight links from head to tail model. Each left and right leg of the front has three DOFs that can determine the direction of the straight walk. Each DOF has two rear legs. Their movement is more passive than the robot's movement front legs. After building the CPG based locomotion, the results of the CPG model are implemented in 3D animation under the SimMechanics 
of First Generation environments, such as in [16]. In order to verify the legged locomotion based on CPG model in 3D animation simulation, the CPG model is embedded on the robot salamander microcontroller using 32 bit Arduino Due. The robot is tested on the straight walk gait as in $3 \mathrm{D}$ simulation.

\section{Salamander Robot Model}

\section{II.1. 3D CAD Design}

In this paper, the development of 3D CAD model on the salamander like- robot has been conducted in parallel with robotic prototype assembly. 3D CAD design of the salamander like-robot model has been performed using SolidWorks computer-aided design (CAD) software. The SolidWorks CAD software has been selected because of its ease of use. The 3D CAD model and the prototype of salamander like-robot that have been developed can be shown in Fig. 1. The dimension of the salamander robot can be seen in Fig. 2.

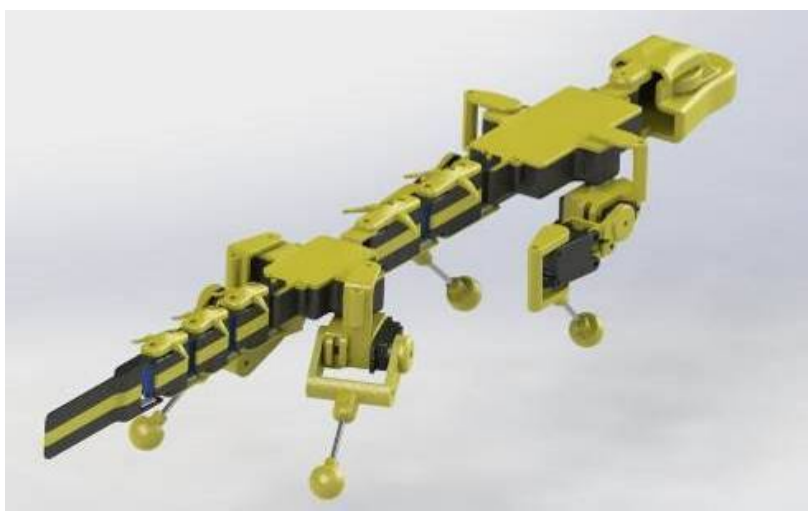

Fig. 1. 3D CAD assembly of the proposed $18 \mathrm{DOF}$ salamander robot

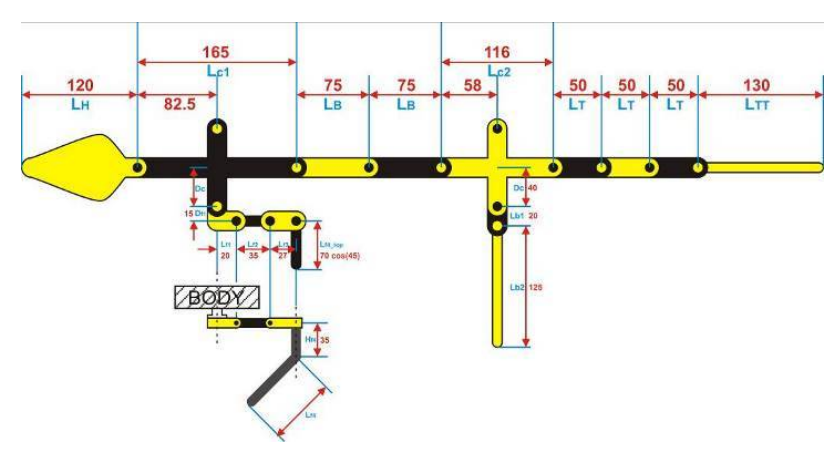

Fig. 2. The dimension of the body links in the salamander robot (all units are in $\mathrm{mm}$ )

It can be seen that the dimensions of the body part in the front legs and the rear legs are not similar. The forelimbs of the front legs have been used for the controller's place and the rear legs have been used for the battery's place. The result of 3D salamander robot model from CAD software as shown in Fig. 1 has been exported in SimMechanics block diagram under MATLAB/Simulink environment using SimMichanics Link plugin. The plugin can be downloaded freely from the Mathworks website. The results of SimMechanics block diagram will be used as 3D animation of salamander robot motion. The 3D animation will visualize the motion of salamander robot on straight walk in 3D view environment. SimMechanics toolbox from MATLAB/Simulink software is a powerful tool for $3 \mathrm{D}$ animation purpose of a mechanical system. It can simulate the motion of mechanical system by giving it with the joint input in 3D view environment. SimMechanics 3D animation had been successfully developed for 3D virtual of five DOF robotic hand on the previous works [17], [18]. The 3D animation of robotic hand can be driven by giving the revolute joint angle inputs for each finger in SimMechanics block diagram.

This salamander like-robot model for 3D animation has used revolute joints to model the joint body of the proposed salamander robot in SimMechanics.

\section{II.2. Gait Cycle for Straight Walking Movement}

In Fig. 1, there is a shaft supporting the body of a salamander robot model between the two joints of the body and the front legs. This consideration is taken because the point serves as the front Central Pattern Generator (CPG) which becomes the reference on the straight walk. The point/CPG in the salamander robot model does not move against time. The kinematics equation of motion (EOM) is developed based on CPG legged locomotion, starting from the head to tail tip.

When walking, the salamander form S-shape standing wave with a length of $1 \lambda$ from head to tail tip. Two points that become the reference when performing straight walking movement are the center point of the connecting body of the front legs (front CPG) and the center point of the rear legs connecting body (rear CPG).

The movement of the front legs adjusts the body movements of the robot salamander. While the front body of the salamander robot leans to the right, the left front leg goes forward. When the front body of the salamander robot leans to the left, the left front leg retreats as it moves forward. The rear leg movement is the opposite movement of the front leg movement. When the left front leg steps back, the rear right leg steps back simultaneously, while the rear left leg moves with the tip of the foot forward. Both the front legs and rear legs have the same distance step. This same step makes the salamander robot to walk straightforward.

\section{CPG Based Locomotion Model}

In this section, the straightforward walk kinematics motion of the salamander robot is developed to determine the angles formed by each joint on the head, body, legs, and tail of the salamander robot. The front CPG has been used as a reference to the straightforward walking movement of salamander robot. The joint angles on the head, body, and tail can be shown by Fig. 3. The body angle on the front CPG has oscillated from $\theta_{\mathrm{C} 1}$ to the centerline, as shown in Fig. 3. 


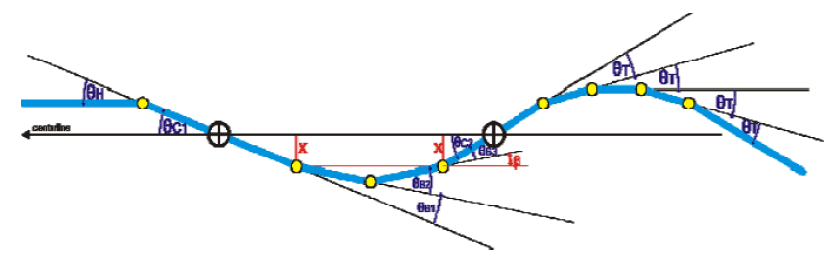

Fig. 3. Joint angles of the salamander robot

The initial angle of $\theta_{\mathrm{C} 1}$ is $0^{\circ}$, so it can be written in a sinusoidal equation using the parameters shown in Fig. 2, and it can be expressed by equation (1):

$$
\begin{aligned}
& \theta_{C 1}=\theta_{C 1(\max )} \sin (\omega t) \\
& d_{f}=D_{C} \cos \theta_{C 1}+L_{f} \cos \alpha+ \\
& -L_{f 4(\text { top })} \cos \left(\theta_{f(\max )}-\gamma_{1}\right) \\
& \alpha=\theta_{C 1}+\theta_{f 1}-\theta_{f 1(\max )} \\
& S=D_{C} \sin \theta_{C 1}+L_{f} \sin \alpha+L_{f 4(t o p)} \sin \left(\theta_{f}-\gamma_{1}\right) \\
& S-S_{0}=D_{C} \sin \theta_{C 2}+ \\
& +\left(L_{b 1}+L_{b 2} \cos \theta_{b 2}\right) \sin \left(\theta_{C 2}-\theta_{b 1}\right)
\end{aligned}
$$

\section{III.1. Head Movement $\left(\theta_{H}\right)$}

The head of the salamander robot is always facing forward, in the other words the head angle $\theta_{\mathrm{H}}$ is always parallel to the centerline. Then the angle on the joint of the head must be opposite direction and equal to $\theta_{\mathrm{C} 1}$. The angular equation for the head joint can be written as in equation (2):

$$
\theta_{H}=-\theta_{C 1}
$$

\section{III.2. Body Movement $\left(\theta_{C 2}, \theta_{B 1}, \theta_{B 2}, \theta_{B 3}\right)$}

The length of the two joints in front body $L_{C 1}$ and the length of two joints on the rear body have the same distance to the centerline. The value of $\theta_{\mathrm{C} 2}$ can be defined as expressed in equation (3):

$$
\sin \theta_{C 2}=\frac{L_{C 1}}{L_{C 2}} \sin \theta_{C 1}
$$

The abdomen of the salamander robot forms a half wave. There are four links on the abdomen. In order to form a half-wave, the joint in the middle must be the peak of the wave. The second and third links have the opposite angle of the direction and the same magnitude towards the centerline that is half of $\theta_{C 1}, \theta_{B 1}$ can be defined as in Equation (4):

$$
\theta_{B 1}=-\frac{1}{2} \theta_{C 1}
$$

From equation (4), the joint angles of $\theta_{\mathrm{B} 2}$ and $\theta_{\mathrm{B} 3}$ can be calculated using equation (5), and (6):

$$
\theta_{B 2}=-\theta_{C 1}
$$

$$
\theta_{B 3}=\theta_{C 2}-\theta_{B 1}
$$

\section{III.3. Tail Movement $\left(\theta_{T}\right)$}

In the straight walking motion, the salamander robot 's tail forms half wave opposing to its body. In the CPG based locomotion model, there are four links for the tail system. The rear leg connecting body and tail tip links forms an equal angle to the centerline and opposites to each other. The magnitude of the joint angle on the tail for the motion of straight walking is equal, it can be defined as written in equation (7):

$$
\theta_{T}=-\frac{1}{2} \theta_{C 2}
$$

\section{III.4. Front Leg Movement}

The straight walking pattern strategy for the front leg is the end of the link always following the offset of the centerline with the $d_{f}$ distance obtained according to the $\theta_{\mathrm{f} 1}$ value and determined from the front leg position at the maximum $\theta_{\mathrm{C} 1}$. The value of $\theta_{\mathrm{f} 1}$ is predetermined and it can vary between $0^{\circ}-\theta_{\mathrm{f} 1}(\max )$. The front leg tip is always following the offset of the centerline with the distance $\mathrm{d}_{\mathrm{f}}$ by adjusting the value of $\theta_{\mathrm{f}}$. The value of $\theta_{\mathrm{f}}$ is range from $0^{\circ}$ to $90^{\circ}$. From the description above and from Fig. 4, the kinematics motion can be written for the front leg as follows:

$$
L_{f}^{2}=D_{f 1}^{2}+\left(L_{f 1}+L_{f 2}+L_{f 3}\right)^{2}
$$

$$
\theta_{f 1(\max )}=90^{\circ}-\gamma_{2}
$$

$$
\alpha=\theta_{C 1}+\theta_{f 1}-\theta_{f 1(\max )}
$$

$$
\begin{aligned}
d_{f} & =D_{C} \cos \theta_{C 1}+L_{f} \cos \alpha+ \\
& -L_{f 4(\text { top })} \cos \left(\theta_{f(\max )}-\gamma_{1}\right)
\end{aligned}
$$

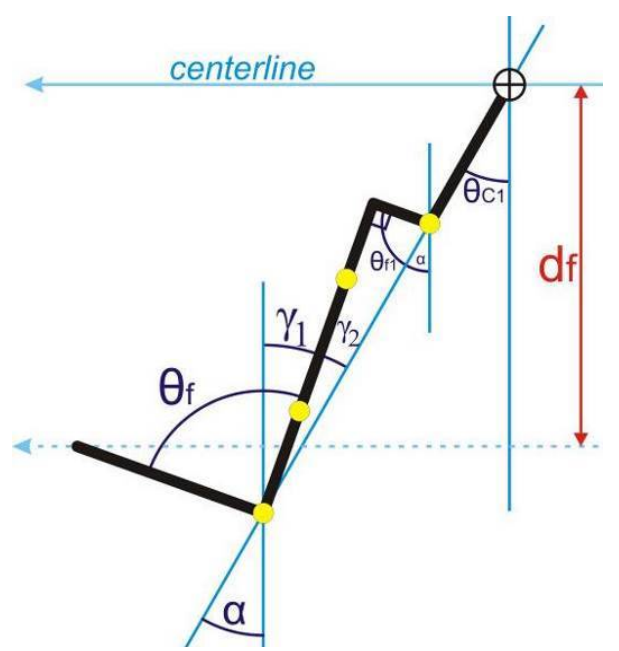

Fig. 4. Front leg movement 
Before presenting the equation of motion on the rear legs, it should be noted that forward strokes on the front legs and rear legs go simultaneously and equal in length, so that no slip conditions occur. $\mathrm{S}_{0}$ is the stroke at the initial position and $\mathrm{S}$ is the actual stroke as shown in Fig. 5. The equation of foot strokes can be written as in equation (5):

$$
S=D_{C} \sin \theta_{C 1}+L_{f} \sin \alpha+L_{f 4(t o p)} \sin \left(\theta_{f}-\gamma_{1}\right)
$$

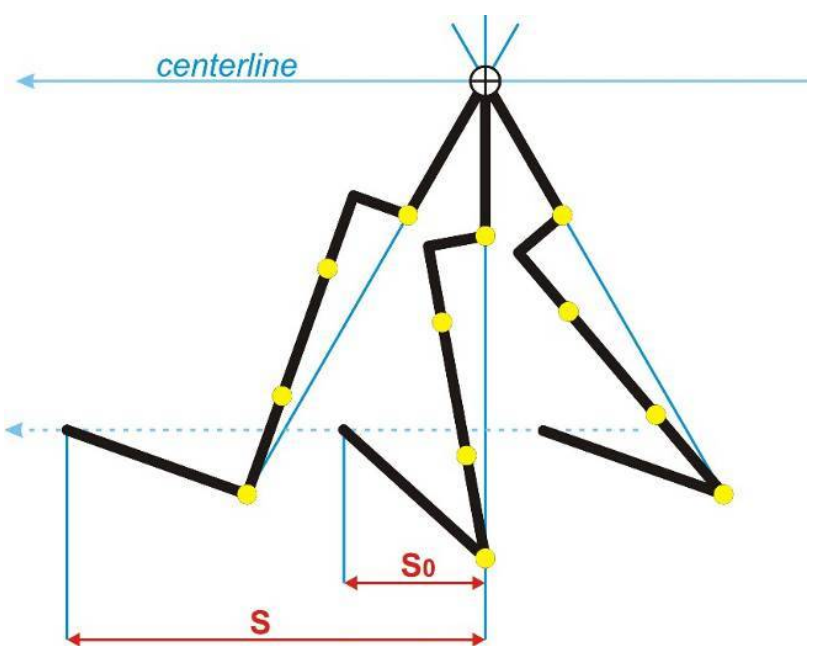

Fig. 5. Front leg stroke

\section{III.5. Front Leg Movement}

The stroke on the rear leg adjusts to the front foot. In Fig. 6, the starting position of the rear foot opposites to the front foot because when it touches the ground, the front, and the rear legs are in opposite position. The equations of the rear leg motion can be written as in equation (12):

$$
\begin{aligned}
& S-S_{0}=D_{C} \sin \theta_{C 2}+ \\
& \quad+\left(L_{b 1}+L_{b 2} \cos \theta_{b 2}\right) \sin \left(\theta_{C 2}-\theta_{b 1}\right)
\end{aligned}
$$

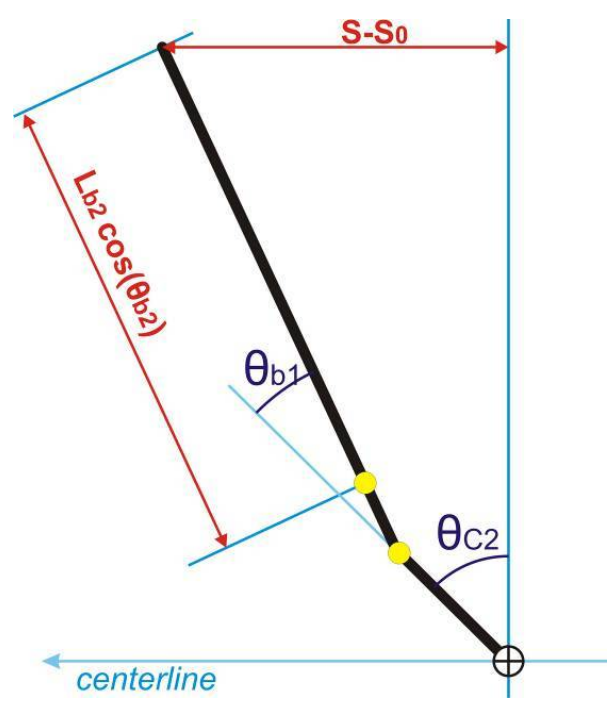

Fig. 6. Rear leg movement

\section{Salamander-Like Robot Prototype}

The prototype of a salamander like-robot has been developed using 3D printing technology. The body of the robot has been printed by using polylactic acid (PLA) material. The main part of the salamander robot has been printed using a 3D delta type printer with a nozzle diameter of $0.2 \mathrm{~mm}$, with a maximum width of $17 \mathrm{~cm}$ and a maximum height of $22 \mathrm{~cm}$. Two servomotors, servo Tower Pro MG995 and Tower Pro SG90 have been used on this salamander-like robot as shown in Figs. 7.

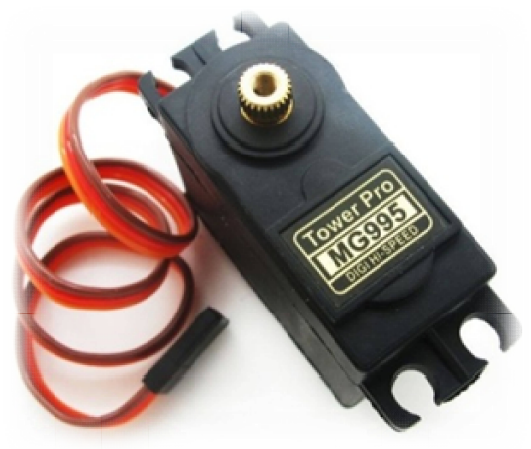

(a)

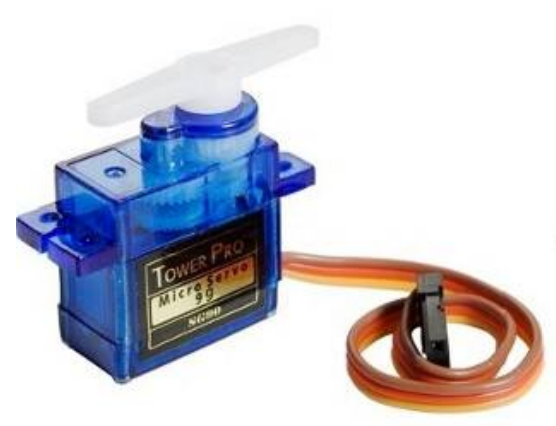

(b)

Figs. 7. The utilized servo motors on salamander like-robot, (a) Tower Pro MG 995 (b) servo Tower Pro SG90

The Servo Tower pro MG995 has a torque varying from $9.4 \mathrm{~kg} \mathrm{~cm}$ at $4.8 \mathrm{~V}$ to $11.00 \mathrm{~kg} \mathrm{~cm}$ at $6 \mathrm{~V}$. This metal gear servo has the mass of $55 \mathrm{~g}$ and it can rotate from $0^{\circ}$ to $180^{\circ}$. This servo has been used for lift motion on the robot's leg because it had a large enough torque to lift the body load of the salamander robot, while Servo Tower Pro SG90 has a torque of $1.8 \mathrm{~kg} \mathrm{~cm}$ and the dimension is quite small that is $22.2 \times 11.8 \times 31 \mathrm{~mm}$. This servo has been used for the vertical axis joint motion of the salamander like-robot body because it has not been affected by gravity and has small dimension. Since the CPG-based locomotion on this robot has required complex and heavy computation, the Arduino Due microcontroller has been selected and used in this study. Arduino Due is a project board variant of the Arduino microcontroller that uses the Atmel SAM3X8E ARM Cortex-M3 CPU. Arduino Due is the first Arduino Development Board to use a 32-bit ARM processor. Development board has a GPIO of 54 pins (12 of which 
are PWM pins), 12 analog pins, 4 UART / serial port hardware, clock count / clock with $84 \mathrm{MHz}$ frequency, USB OTG connection, 2 DAC (digital-to-analog converter), 2 TWI (Two Wire Interface, compatible with I2C protocol from Phillips), standard power supply jack (5.5 / $2.1 \mathrm{~mm}$ ) jack, SPI header connector, JTAG header connector, reset button, and an erase button). The drawback of the Arduino Due compared to other Arduino variants that use MCU Atmega is the absence of integrated EEPROM. In order to control the salamander like-robot using Arduino Due only the general purpose input/output (GPIO) pin is needed because in this early research the remote control has not been used. The final prototype of the built salamander like-robot is shown in Fig. 8. The robot has nine joints with eight links from head to tail. The nine joints used metal gear servo Tower Pro SG90. Each left and right of the front leg has three DOF while each rear leg has two DOF because of its more passive movements than the robot's front legs. The joints on both front and rear legs have utilized metal gear servo Tower Pro MG995.

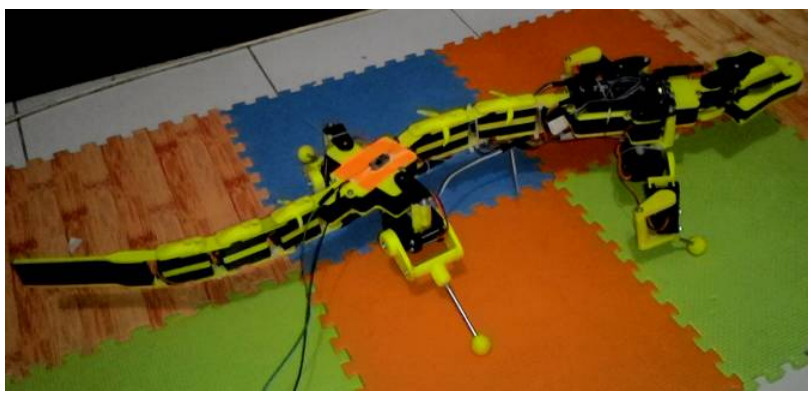

Fig. 8. The resulted salamander-like robot

\section{Results and Discussions}

In this section, the kinematics motion based on the developed CPG based legged locomotion is implemented in simulation under virtual reality and real salamander like-robot to walk in straight walk motion. The 3D animation in virtual reality is conducted using SimMechanics First Generation feature in MATLABSimulink software. For the embedded programs on the prototype of salamander like-robot, Simulink Support Package for Arduino Hardware has been used

\section{V.1. Straightforward Walk in Simulation and Virtual Reality}

The SimMechanics - First Generation toolbox under MATLAB/Simulink environment has been chosen because it is simpler and it conforms to the problem limitation of not discussing robot dynamics, only its kinematics. The $3 \mathrm{D}$ virtual reality model of the salamander robot has been imported from * .xml and * .stl files created by exporting the robot model in Solidworks CAD software assemblies using the Sim Mechanics Link plugin. When the * .xml file has been imported using the 'mechimport' command function in
MATLAB, the software automatically generates the auto-generate block diagram of the salamander robot in the Simulink environment according to the Solidworks assembly. The diagrams formed need to be tidied up in order to remember the many parts and joints to make it easier in the next programming of the block diagram in Simulink. The joint angles of salamander robot are determined using equation from (1) to equation (13). The results of joint angles are presented by the corresponding plot from Fig. 9 to Fig. 13.

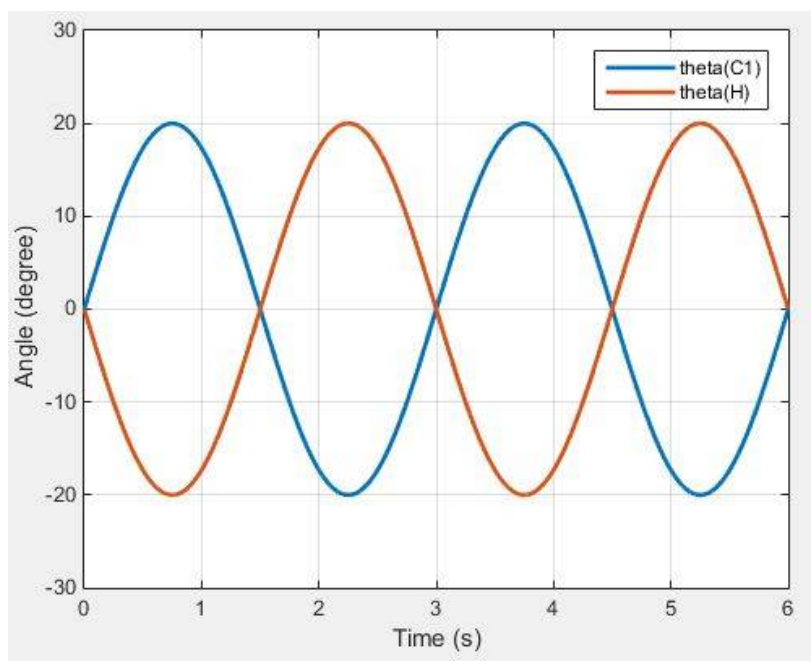

Fig. 9. Predefined joint angle inputs of the head and front CPG

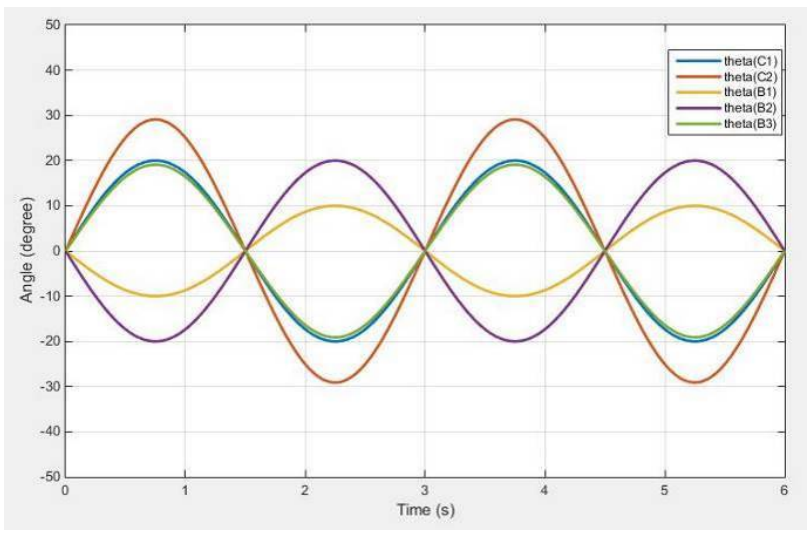

Fig. 10. Predefined joint angle inputs of CPG and body

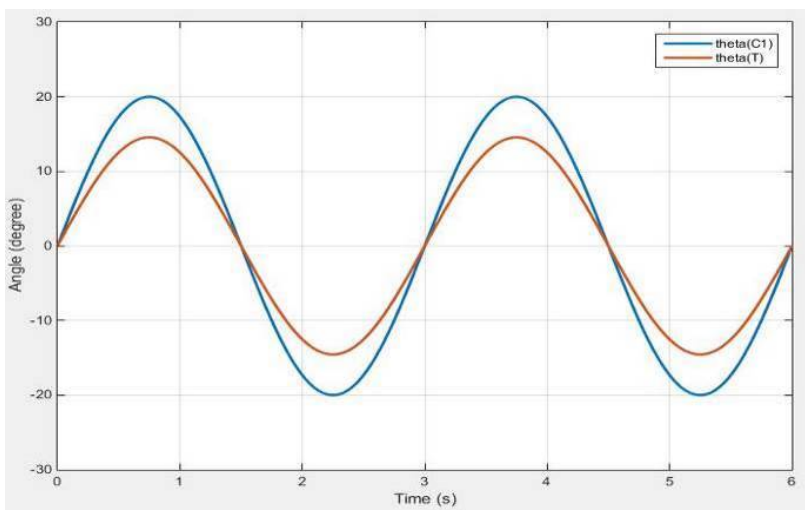

Fig. 11. Predefined joint angle inputs of front CPG and tail 


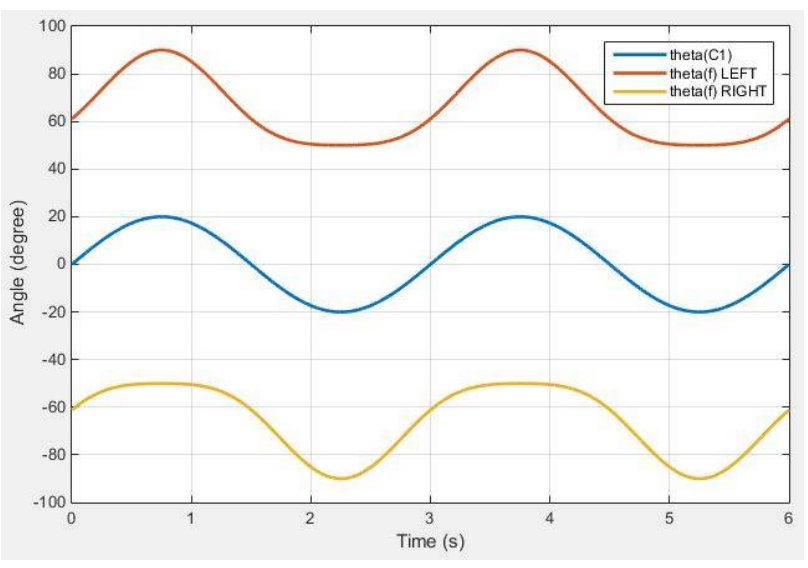

Fig. 12. Predefined joint angle inputs of front CPG and front leg

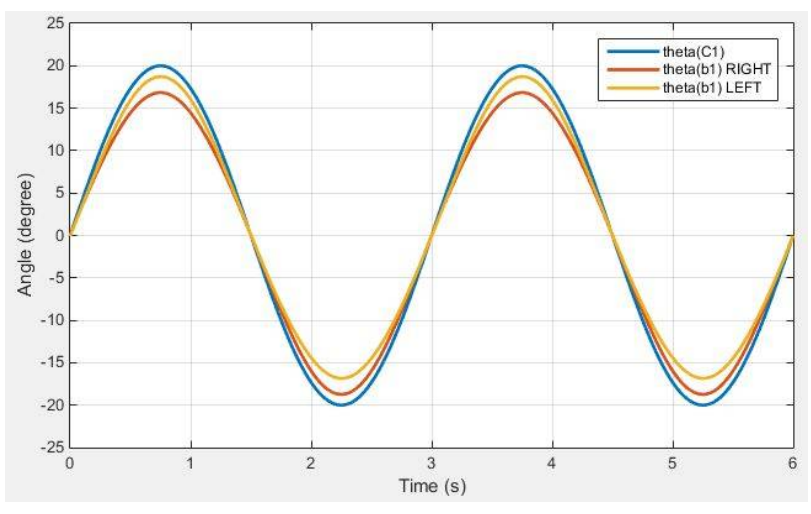

Fig. 13. Predefined joint angle inputs of front CPG and rear leg

Fig. 9 shows $\theta_{\mathrm{C} 1}$ and $\theta_{\mathrm{H}}$ corresponding to the equation (2), the value of $\theta_{\mathrm{H}}$ is opposite to $\theta_{\mathrm{C} 1}$. The joint angles in the salamander' body, front $\mathrm{CPG}$, and rear CPG resul from equation (4) to equation (6) as shown in Fig. 10.

The resulted joint angle motion in tail can be presented as in Fig. 11. The joint angle in the tail is less than the joint angle in front CPG. The joint angles in rear legs and front legs are presented in Fig. 12 and Fig. 13 respectively.

The motions of joint angle inputs from Fig. 9 to Fig. 13 are simulated using SimMechanics 3D Animation for the straight walk motion on the 3D model of salamander robot. The result of 3D animation simulation in SimMechanics environment after given equation of motion input can be seen in Fig. 14. From the simulation results in $3 \mathrm{D}$ virtual reality of the salamander robot motion, the robot model has succeeded in forming a curve that resembles standing wave with the head always forward. The front leg always moves parallel to the centerline line when it has been in contact with the ground. Rear leg stroke has been in accordance with the front leg stroke opposite side. Every 1.5 second the salamander body becomes straightforward. These results of $3 \mathrm{D}$ animation motion of the robot have shown that the developed model of CPG based legged locomotion can be always forward. The front leg always moves parallel to the centerline line when it has been in contact with the ground. Rear leg stroke has been in accordance with the front leg stroke opposite side. Every 1.5 second the salamander body becomes straightforward. This result of $3 \mathrm{D}$ animation motion of the robot shows that the developed model of CPG based legged locomotion can be implemented to drive the proposed salamander robot on straight walk conditions.

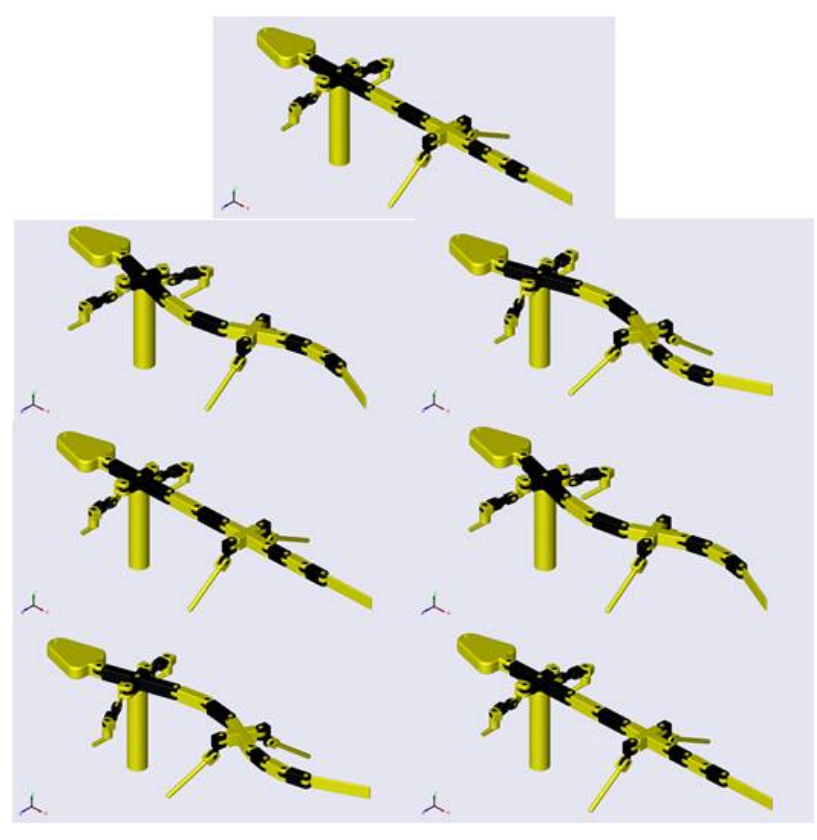

Fig. 14. The sequence of motion of salamander robot in SimMechanics 3D animation from $1 \mathrm{~s}$ to $6 \mathrm{~s}$

\section{V.2. Straight Walk in the Real Salamander-Like Robot}

In order to verify the effectiveness of the developed CPG based locomotion model in virtual reality simulation as presented in section V.1, the CPG model has been verified by using embedded CPG in a real salamander-like robot. The kinematics equation developed from CPG based legged locomotion as presented in equation (1) through equation (13) has been developed using block diagram under MATLAB Simulink environment. After the CPG based locomotion model as in equation (1) through (13) has been built in Simulink block diagram. The resulted motions of joint angle inputs from Fig. 9 to Fig. 13 is utilized as joint motion input in servo motor command of the salamander like-robot prototype as shown in Fig. 8. The command of servomotor has been implemented using Standard Servo Write block. The block diagram of CPG model has been embedded in Arduino Due using Simulink Support Package for Arduino Hardware. The model run with the sampling rate of $50 \mathrm{~Hz}$. The Arduino Due microcontroller can run the CPG model in real time without overrunning in computation. The overall block diagram CPG equation of straight walk motion from equation (1) to equation (13) was developed using MATLBA/Simulink software. The block diagram shown in Fig. 15 has been embedded into Arduino Due microcontroller. 


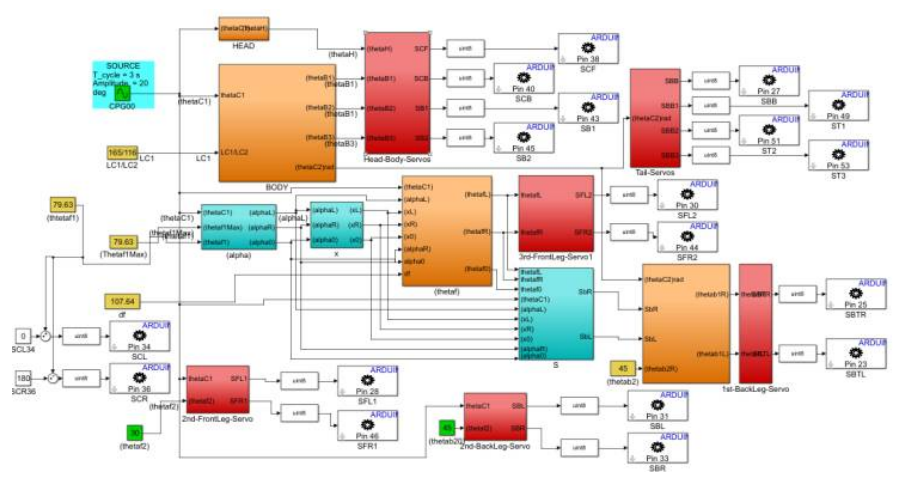

Fig. 15. Developed CPG equation of motion block diagram in MATLAB/Simulink environment

Based on the experimental results, the proposed salamander robot prototype has succeeded in forming $\mathrm{S}$ curve that resembles a standing wave with the head always forward on the straightforward walk. The motion of the salamander robot's body links had similar motion as shown in virtual reality simulation results.

In the experimental result, the front leg has always moved parallel to the centerline line when the foot has been in contact with the ground. Rear leg stroke has been in accordance with the front leg stroke opposite side. Both in virtual reality simulation and experimental had the same initial condition i.e. 0 degree. Every 1.5 second, the salamander body becames straight both in the virtual reality simulation and experiment. Based on the legged locomotion result in the experimental work, the developed CPG based locomotion had been verified that the salamander like-robot can walk effectively in straight walk as depicted in Fig. 16.

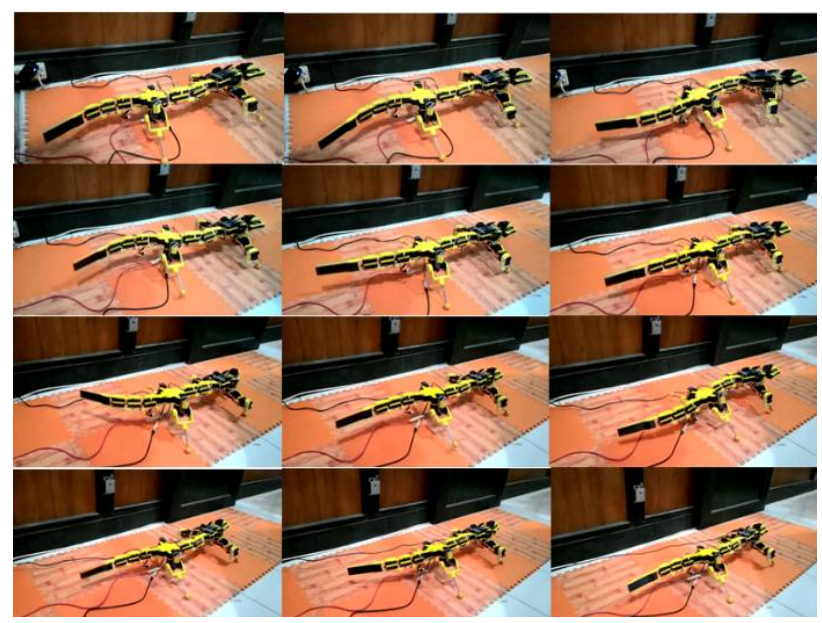

Fig. 16. The sequence of images of the salamander-like robot walking straightforward

\section{Conclusion and Future Work}

The proposed salamander like-robot uses CPG approach by dividing the salamander robot body into smaller segments that can represent the segmentation function of the actual salamander body. The CPG based locomotion of the spinal cord of the salamander like- robot is represented by eight joints with nine links from head to tail model. The effective CPG based legged locomotion for 18 DOF salamander like-robot has been successfully developed and implemented in both virtual reality simulation and experimental work. Based on the simulation, the predefined joint angle inputs in salamander robot can be utilized to drive the robot to do straight walk motion that can mimic the walking of a real salamander naturally. Based on the legged locomotion result in experimental work, the developed CPG based locomotion has verified that the salamander like-robot can walk straight effectively. The research has succeeded in the developing of a low-cost 18 DOF salamander likerobot using 3D printing technology that can walk naturally like a salamander. In future research, the motion of the salamander robot in turn right and turn left will be developed and embedded into the proposed salamander robot. After the CPG based locomotion is embedded into the robot, it will be used as a low-cost amphibious robot that can be implemented to support Search and Rescue (SAR) especially in disaster areas such as flood, earthquake, tsunami, or landslide

\section{References}

[1] Ijspeert, Auke Jan, Jean-Marie Cabelguen, Gait Transition from Swimming to Walking: Investigation of Salamander Locomotion Control Using Nonlinear Oscillators, In: Kimura, Hiroshi, Kazuo Tsuchiya, Akio Ishiguro, Hartmut Witte. (eds.) Adaptive Motion of Animals and Machine, 2005, pp. 177-186

[2] Horvat, T., K. Karakasiliotis, K. Melo, L. Fleury, R. Thandiackal and A. J. Ijspeert. Inverse kinematics and reflex based controller for body-limb coordination of a salamander-like robot walking on uneven terrain. IEEE/RSJ International Conference on Intelligent Robots and Systems (IROS), Hamburg, 2015, pp. 195-201

[3] K. Karakasiliotis and A. J. Ijspeert, Analysis of the terrestrial locomotion of a salamander robot, 2009 IEEE/RSJ International Conference on Intelligent Robots and Systems, St. Louis, MO, 2009, pp. 5015-5020.

[4] A. Crespi, K. Karakasiliotis, A. Guignard and A. J. Ijspeert, Salamandra Robotica II: An Amphibious Robot to Study Salamander-Like Swimming and Walking Gaits, in IEEE Transactions on Robotics, vol. 29, no. 2, pp. 308-320, April 2013.

[5] Li, L., Wang, C. and Xie, G., A general CPG network and its implementation on the microcontroller. Neurocomputing, 167, pp.299-305, 2015.

[6] Billah, M.M. and Khan, M.R., February. Bio-inspired snake robot locomotion: A CPG-based control approach. In Information Technology: Towards New Smart World (NSITNSW), 2015 5th 
National Symposium on (pp. 1-6).

[7] Bing, Zhenshan, Long Cheng, Kai Huang, Mingchuan Zhou, and Alois Knoll. CPG-based control of smooth transition for body shape and locomotion speed of a snake-like robot. In 2017 IEEE International Conference on Robotics and Automation (ICRA), pp. 4146-4153. IEEE, 2017.

[8] Wang, Z., Gao, Q. and Zhao, H., 2017. CPG-inspired locomotion control for a snake robot basing on nonlinear oscillators. Journal of Intelligent \& Robotic Systems, 85(2), pp.209-227.

[9] Zhang, Dong, Qing Xiao, Zhengcai Cao, Ran Huang, and Yili Fu. Smooth transition of the CPG-based controller for snake-like robots. In 2017 IEEE International Conference on Robotics and Biomimetics (ROBIO), 2017 pp. 2716-2721.

[10] Rostro-Gonzalez, H., Cerna-Garcia, P. A., Trejo-Caballero, G., Garcia-Capulin, C. H., Ibarra-Manzano, M.A., Avina-Cervantes, J. G. and Torres-Huitzil, C., A CPG system based on spiking neurons for hexapod robot locomotion. Neurocomputing, 170, pp.47-54, 2015.

[11] Barron-Zambrano, J. H., Torres-Huitzil, C. and Girau, B. Perception-driven adaptive CPG-based locomotion for hexapod robots. Neurocomputing, 170, pp.63-78, 2015.

[12] Zhao, W., Hu, Y., Zhang, L. and Wang, L. Design and CPG-based control of biomimetic robotic fish. IET control theory \& applications, 3(3), pp.281-293., 2009.

[13] Hu, Y., Tian, W., Liang, J. and Wang, T. Learning fish-like swimming with a CPG-based locomotion controller. In 2011 IEEE/RSJ International Conference on Intelligent Robots and Systems (IROS), 2011 pp. 1863-1868.

[14] Wang, L., Wang, S., Cao, Z., Tan, M., Zhou, C., Sang, H. and Shen, Z., 2005, December. Motion control of a robot fish based on CPG. In IEEE International Conference on Industrial Technology ICIT 2005, 2005 pp. 1263-1268.

[15] Wang, M., Tang, Z., Chen, B. and Zhang, J. Locomotion control for quadruped robot based on Central Pattern Generators. In 2016 35th Chinese Control Conference (CCC), pp. 6335-6339.

[16] M. Munadi, M. S. Nasir, M. Ariyanto, Norman Iskandar, and J. D. Setiawan, Design and simulation of PID controller for lower limb exoskeleton robot. AIP Conference Proceedings, 2018. Vol. 1983, No.1, pp. 060008.

[17] R. Ismail, M. Ariyanto, W. Caesarendra and A. Nurmiranto, Development of robotic hand integrated with SimMechanics 3D animation, 2016 International Seminar on Intelligent Technology and Its Applications (ISITIA), Lombok, 2016, pp. 629-634.

[18] M. Ariyanto, R. Ismail, A. Nurmiranto, W. Caesarendra, Paryanto and J. Franke, Development of a low cost anthropomorphic robotic hand driven by modified glove sensor and integrated with 3D animation, 2016 IEEE EMBS Conference on Biomedical Engineering and Sciences (IECBES), Kuala Lumpur, 2016, pp. 341-346.

[19] El Hansali, H., Bennani, M., Gait Kinematic Modeling of a Hexapod Robot, (2017) International Review of Mechanical Engineering (IREME), 11 (3), pp. 200-207. doi:https://doi.org/10.15866/ireme.v11i3.10987

[20] Caceres, C., Puerta, J., Jiménez, R., Rojas, D., Design of a BioInspired Equine Robot Prototype, (2016) International Review of Mechanical Engineering (IREME), 10 (1), pp. 12-17. doi:https://doi.org/10.15866/ireme.v10i1.7672

\section{Authors' information}

Department of Mechanical Engineering, Diponegoro University, Semarang, Indonesia.

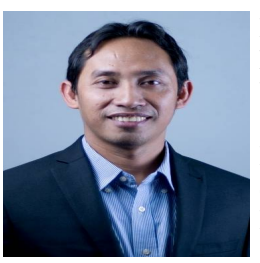

Dr. Eng. Munadi received the B.E. degree in Mechanical Engineering from Diponegoro University, Semarang, Indonesia in 2001. From 2001-2005 he joined Pura Group Corp., Kudus, Indonesia. In 2007, he received the M. Eng. degree from Mechanical Engineering, Bandung Institute of Technology, Bandung, Indonesia. In 2011, he received the Dr. Eng. degree from the Graduate School of Engineering, University of Fukui, Fukui, Japan. He is currently a lecturer in the Department of Mechanical Engineering, Diponegoro University, Indonesia. His current research interests relate to hybrid adaptive and learning controller for robot manipulators, intelligent control, and soft robot.

E-mail: munadi@undip.ac.id

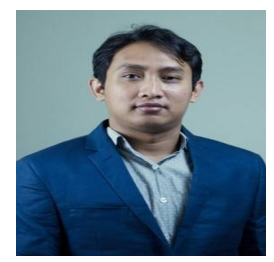

Mr. Mochammad Ariyanto received B.Sc. and M.Sc. degrees from Department of Mechanical Engineering, Diponegoro University, Indonesia, in 2010 and 2013 respectively.

Currently, he is a lecturer at Diponegoro University, Semarang, Indonesia. $\mathrm{He}$ has published more than 35 papers in international journal, book chapter, and conference proceeding. His research interests are biorobotics, mechatronics, soft robot, and intelligent control.

E-mail: mochammad ariyanto@ft.undip.ac.id

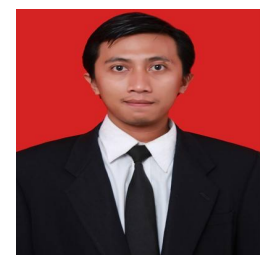

Mr. Kharisma A. Pambudi received B.Sc. degree from Department of Mechanical Engineering, Diponegoro University, Indonesia, in 2016. Currently, he is a master student at Department of Mechanical Engineering, Diponegoro University, Semarang, Indonesia. His research interests are biorobotics, mechatronics, and soft robot.

E-mail: kharismaagungpambudi@gmail.com

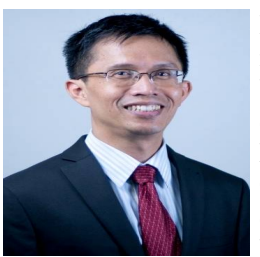

Dr. Joga D. Setiawan received the B.Sc. degree in Mechanical Engineering from Northeastern University, Boston, USA in 1992; and the M.Sc. degree in Aeronautics/Astronautics from Massachusetts Institute of Technology (MIT), Cambridge, USA in 1996. He received the Ph.D. degree in Mechanical Engineering from Michigan State University, USA in 2001. He is currently a lecturer in the Mechanical Engineering Department of Diponegoro University, Indonesia. Dr. Joga has published more than 45 papers of book chapter, international journal, and conference proceedings in the area of control system, intelligent system, and mechatronics. He is member of The Institute of Electrical and Electronics (IEEE)

E-mail: joga.setiawan@ft.undip.ac.id 